\title{
Iron Deficiency Anemia in Children: Authors' Reply
}

\author{
Girish Subramaniam $^{1} \cdot$ Meenakshi Girish ${ }^{2}$
}

Received: 16 August 2015 / Accepted: 9 September 2015 /Published online: 8 October 2015

(C) Dr. K C Chaudhuri Foundation 2015

To the Editor: 'Catch them young' is the mantra when it comes to evaluating red blood cells in the diagnosis of iron deficiency anemia. Young erythrocytes, the reticulocytes, with their shorter life span score over mature erythrocytes when it comes to studying early markers of iron deficiency.

Sidhu and Goyal deserve credit for highlighting this aspect in their correspondence, as a response to our article on iron deficiency anemia [1]. While we too agree that the various reticulocyte indices, reticulocyte hemoglobin content $(\mathrm{CHr})$, reticulocyte maturity index (RMI) and red cell size factor (Rsf) may prove to be better parameters than the conventional ones in the early diagnosis of iron deficiency anemia, we refrained from dwelling on them in our article primarily because the article was meant for general practitioners (Pediatricians in general practice).
We would like to thank Sidhu and Goyal for showing a keen interest in our article and on an issue of great public health importance.

Conflict of Interest None.

Source of Funding None.

\section{Reference}

1. Subramaniam G, Girish M. Iron deficiency anemia in children. Indian J Pediatr. 2015;82:558-64.
Girish Subramaniam

girishsubramaniam25@gmail.com

1 Department of Pediatrics, Midas Children Hospital, Nagpur 440010, India

2 Department of Pediatrics, NKP Salve Institute of Medical Sciences, Nagpur, India 